\title{
Automated Mosaicking of Medical x-ray Images using SIFT Algorithm
}

\author{
Sanjeevakumar Harihar ${ }^{1 *}$, Dr.Manjunath $\mathrm{R}^{2}$ \\ ${ }^{1}$ Research Scholar, Jain University, Bangalore, Asst Prof, ECE Dept, CIT, Gubbi, Karnataka, India \\ ${ }^{2}$ Principle Consultant, Wipro Technologies Ltd, Bangalore, Karnataka, India \\ ${ }^{1}$ sanjeevkumar.harihar@gmail.com, 22
}

DOI: $10.51201 / J U S S T / 21 / 05182$

http://doi.org/10.51201/JUSST/21/05182

\begin{abstract}
Image registration is a process of joining many number of images that have similar overlapping regions of the same scene in order to make panoramic image. In the field of medical, multimedia and image processing applications image registration process stands challenging. The work presented here on medical images which can be applicable for long limb operations and scoliosis operations. Traditional $x$-ray machines produces single frame of $x$-ray image containing a portion of the body part,but it cannotgenerate large view of body $x$-ray image in a single frame.This problem can be solved by creating panoramic image by combining multiple images. The work proposed in this paper can automatically produces panoramic x-ray image by stitching multiple $x$-ray images. The proposed work uses scale-invariant feature transform (SIFT) for mosaicking x-ray images as a local feature point extractor which uses difference of Gaussian (DOG) and invariant to orientation and scale. Based on the location relationship of $x$-ray images, random sample consensus (RANSAC) is incorporated to remove the effect of mismatched point pairs in $x$-ray images and to generate the panoramic view. The performance of the system is computed by using structural and time constraint parameters and are compared with different feature detection techniques. The experimental results shows that combing of SIFT and RANSAC yields less processing time with increase in similarity measures.
\end{abstract}

Keywords: SIFT, DOG, RANSAC, SURF, PCA

\section{INTRODUCTION}

Image registration or image stitching is producing a panoramic view image by registering multiple images that have similar view. A panorama is a large view of scenery. It can be created by joining several pictures that have overlapping regions of the same scene.Image mosaicking plays very vital role in the field of satellite image processing, in medical domain requires mosaicking of radiographyimages, in x-ray image processing where whole body to be viewed. The large view of x-ray images helps doctors to diagnose the disease like hand alignment, leg 
alignment etc. Traditional x-ray machines produces single frame of x-ray image containing a portion of the body part, but it cannot generate large view of body $\mathrm{x}$-ray image in a single frame. This problem can be solved by creating panoramic image by combining multiple images. Basically image mosaicking can be categorized into two major groups. They are feature extraction based method and direct method. A pixel to pixel mismatching can be reduced by direct method [1].Whereas in the other hand feature based methods extracts the distinctive unique features and then matches similar regions present in two images using the identified features. The feature detectors are robust which scale, rotation are and translation invariant can identify the salient features. There has been a rapid growth in medical image stitching using feature based approach. Different feature based detectors are namely Scale Invariant feature Transform (SIFT) [2], Harris [4] [5] detector, Speedup Robust Feature detector (SURF) [3], (PCA-SIFT), Principal Component Analysis SIFT [6]. Depending on the nature of the problem appropriate detectors are chosen. After detecting the features, feature matching, homography estimation and images stitching is performed based on the location relationship of two images. The paper is organized in five parts. Part 2 reveals the background survey. Part 3 provides the proposed imagemosaicking methodology. Part 4, provides experimental results. Part 6, discusses the conclusion.

\section{Literature Review}

Many researchers had been working on image mosaicking using feature extraction based techniques. They are developing different feature based techniques that can detect reliable and redundant features than the existing techniques. But still there is challenge in applications like medical and satellite image processing domains. There are many feature based detectors are used in image mosaicking like SIFT, Harris detector and SURF.

SIFT algorithm is proposed in 1999 by David G. Lowe of the British University of Columbia and was improved [7 ,8] in 2004.SIFT is widely used as a matching algorithm based on features since it exhibits invariance to scaling, rotation, translation and robustness to illumination change [8,9]. SIFT is used as an important feature technique in the field of medical domain as it achieves good results in medical image mosaicking.

From the past 15 years SIFT algorithm is used as one of the most widely used and efficient feature extraction methods in image processing. SIFT has been improved in two aspects namely feature point extractor and feature point matching. Principal component analysis (PCA) and Independent component analysis (ICA) algorithms are used with feature point extractor in order to reduce the feature dimension there by increasing the speed [10,11] of the SIFT algorithm.

Based on the Literature, image stitching are mainly categorized into two techniques namely direct based and feature based approaches. Direct based approach involves measuring the camera attributes in order to minimize the errors parameters created due to intensity variations at the overlapped regions. Based on the variations at these regions a Transformation matrix is evaluated. Direct based approach provides accurate registration, but they fail image contrast, intensity variations and noise in the images.

Feature based approach identifies the feature points like points, corners, lines, edges or other shape based features and evaluates the relationship between these features.Compared to direct based approach feature based approach is 
robust to illumination change, scale, noise and orientation change in the image. It calculates the features around the local region to describe feature descriptor. In this approach, image comparison is done with features in one image with features generated in the other image using the feature descriptors [12].

There are many diagnostic methods used in medical imaging systems. Behavior-based approach is ideal for light change, scale, noise, and direction change in the image. This method is very fast, and has the ability to identify a panorama image by examining the neighbor's connection between two images. However, feature-based techniques rely on accurate recognition of image features.

Numerous studies have been conducted on the approach to medical image registration. Cross-linking is often used because it uses FFT (Fast Fourier Transform) to record two images of the same size. But it is not cost-effective. [13]

Using Feature based image registration has seen as great move. The mostly used detectors are the SIFT,Harris and SURF [14].Gong imaging technique is presented. SURF detector used in feature list. The weak point is that it is not possible to distinguish the correct characteristics in the image by sound [4] [5]. Singla combined SIFT and SURF [15] to produce stitched images using X-ray images. However, RANSAC (Random Sample Agreement) algorithm was used to select the correct behavior points.

\section{METHODOLOGY}

SIFT algorithm exhibits the property of difference of Gaussians provides the difference of Gaussians (DOG) scalespace. SIFT extracts the feature points, and removes the unstable feature points; identifies the feature point's principal direction, generates the feature descriptor on the various images having multi spatial scales, thereby provides robust to illumination change, scale, noise and orientation change [7].

The logic behind success is to obtain visual quality information under a number of dimensions, directions, and to analyze the essential features of these images through general analysis. [7] In Measurement $\sigma$, we can combine the function of $\mathrm{G}(\mathrm{x}, \mathrm{y}, \sigma)$ to establish the dimensions of image (x, y, $\sigma)$, which is abbreviated to L. SIFT To maximize the performance of characteristic scores, the Gauss have been referred to as $\mathrm{D}(\mathrm{x}, \mathrm{y}, \sigma)$, and $\mathrm{D}(8)$ as the measurement area $\mathrm{L}(\mathrm{x}, \mathrm{y}, \sigma)$.

Each image in the DOG scale D of I (x,y) is the same as the eight adjacent pixels of each pixel and the adjacent images of the two recent scales.Feature point is considered based on the value (minimum or maximum) at this pixel.However, we will get onlyoffset at locations of the feature point, we need to eliminate the feature point having lower contrast [8].In order to get precise location $\mathrm{x}^{\wedge}$ of feature point A' we use the Taylor series expansion function $\mathrm{D}$ at point $\mathrm{A}$ as given in equation 1 .

$$
D=D_{A}+\frac{\partial D_{A}{ }^{T}}{\partial x} x+\frac{1}{2} x^{T} \frac{\partial^{2} D_{A}}{\partial x^{2}} x
$$


Where $\mathrm{x}=(\mathrm{x}, \mathrm{y}, \sigma)^{\mathrm{T}}$ is the offset from point A to point $\mathrm{A}^{\prime}$. To obtain the exact point [8] $\mathrm{x}$ of the other characteristic, point A' Lowe. Evaluate the derivatives on both sides of D (x) as given in Equation (2) and calculate the 'DOG scale-space function $\mathrm{D}(\mathrm{x}) \mathrm{A}^{\prime}$ in Equation (Equation (3)).

$$
\begin{aligned}
& \hat{x}=-\frac{\partial^{2} D_{A}{ }^{-1}}{\partial x^{2}} \frac{\partial D_{A}}{\partial x} \\
& D(\hat{x})=D_{A}+\frac{1}{2} \frac{\partial D_{A}{ }^{T}}{\partial x} \hat{x}
\end{aligned}
$$

In the DOG space, the parameter Dc is considered as the threshold contrast and is used to measure the feature point stability [8].Here, the value of Dc is set to 0.03 and the feature point whose value is lesser than 0.03 are eliminated [10].Many researchers found that, default selection Dc for all images leads to a problem resulting in lower accuracy in feature point extraction [16] and lower efficiency. Hence, for different types of medical images we need to consider the appropriate value for Dc to get accurate feature points.

\section{Removal of Mismatched Points}

The function descriptors extracted in the image mosaic using the SIFT algorithm generate a large number of pairs of unadjusted points, leading to a decrease in the mosaic's performance [17]. The corresponding precision can be increased using the algorithm of the random sampling algorithm (RANSAC), which has the great potential to identify [18] strongly matched pairs. The RANSAC algorithm was proposed by Fischler and Bolles in 1981 and was able to eliminate unwanted values in a set of inspection data through parameters of mathematical models [19]. If we want to obtain good results, we need to investigate continuous calculations to improve the efficiency of the RANSAC algorithm, which results in a greater number of simulations [20, 21]. In other words, it is necessary to integrate a simple method to remove an incompatible detection application using medical radiation.

\section{RESULTS}

This work presents image mosaicking system implemented on medical x-ray images. The proposed work performs image stitching based on feature based approach.SIFT algorithm is used in order to extract the feature descriptors.SIFT based approach is robust to illumination change, scale, noise and orientation change in the image. It calculates the features around the local region to describe feature descriptor. The proposed work is compared with two detectors namely Harris and SURF. The experimental results sows that the proposed system exhibits better accuracy and execution time when compared with other feature detector techniques.Using X-rays, we examined the accuracy of our design. Our input samples include X-ray images of the spine, lower extremities, and upper extremities. As an input image, two overlapping X-rays are needed to expand our proposed system. It takes about $15-30 \%$ of the overlapping regions to expand.

The device proposed for the proposed method is a Core i7 CPU with $2 \mathrm{GHz}$ and $4 \mathrm{~GB}$ of RAM. Table 1 compares the performance characteristics tested by SIFT, SURF and Harris. 


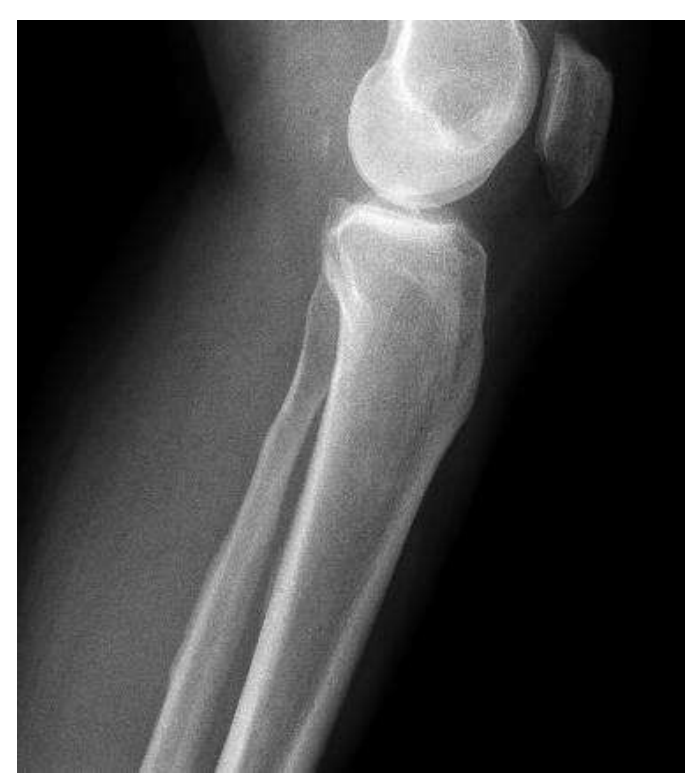

(a) Input image 1

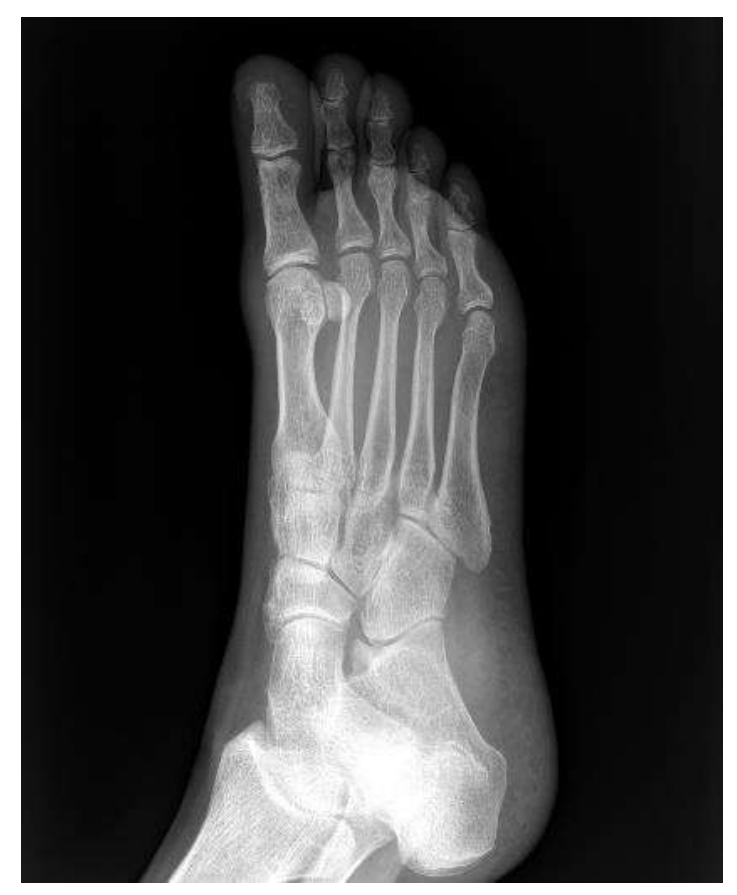

(a) Input image 1

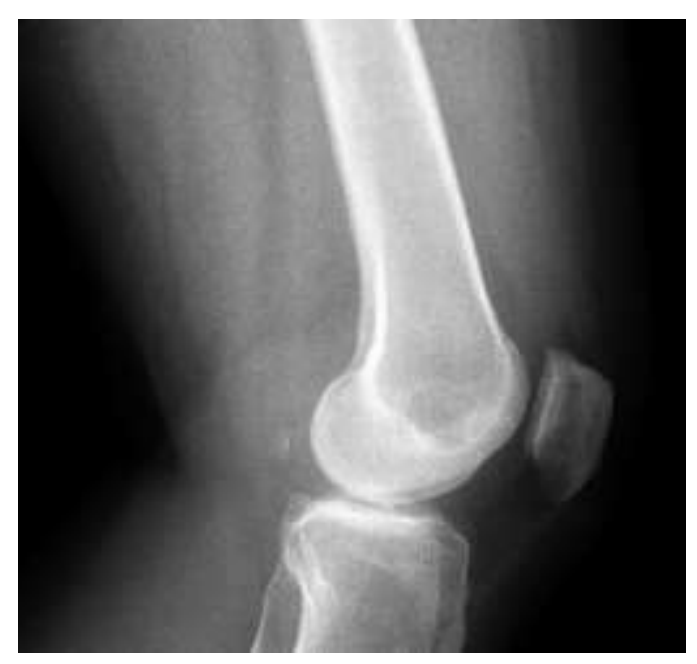

(b) Input image 2

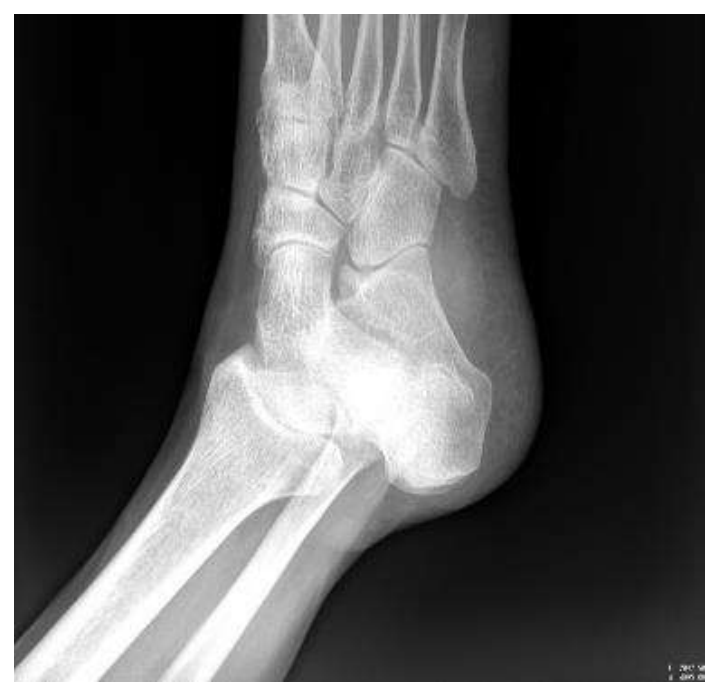

(b) Input image 2

Figure 1. The example input images that used in our proposed system. 


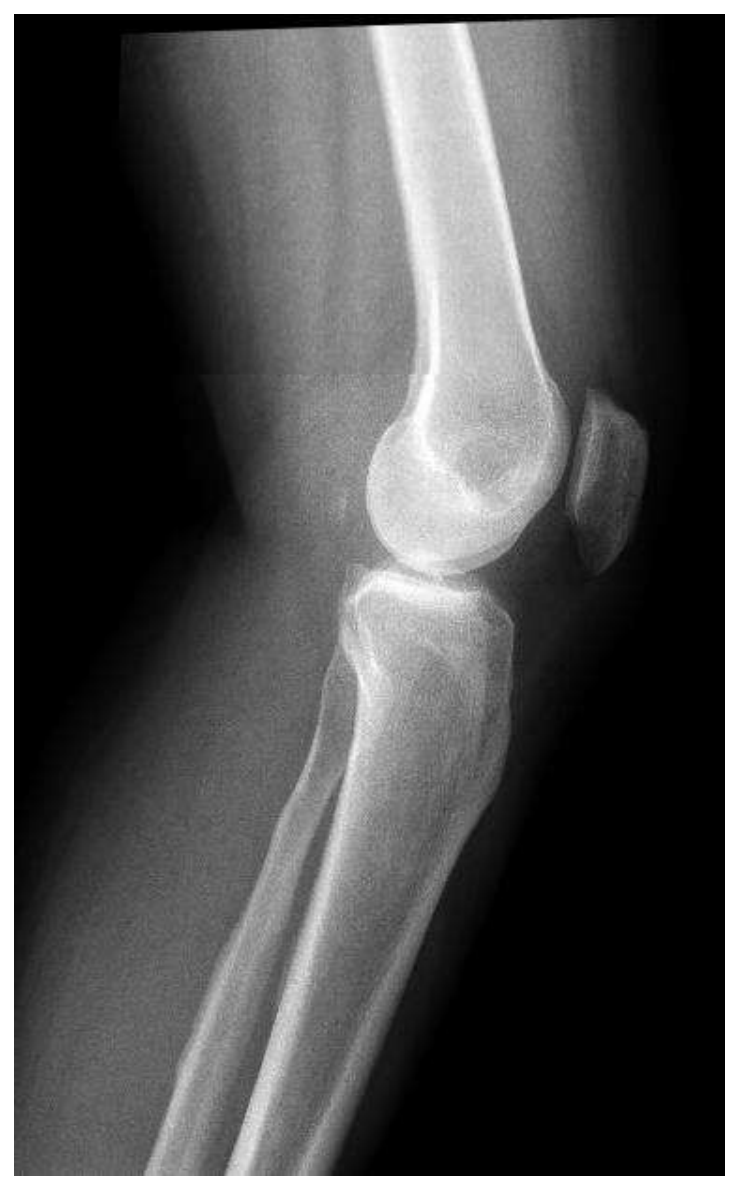

Figure 2. Final knee stitched image

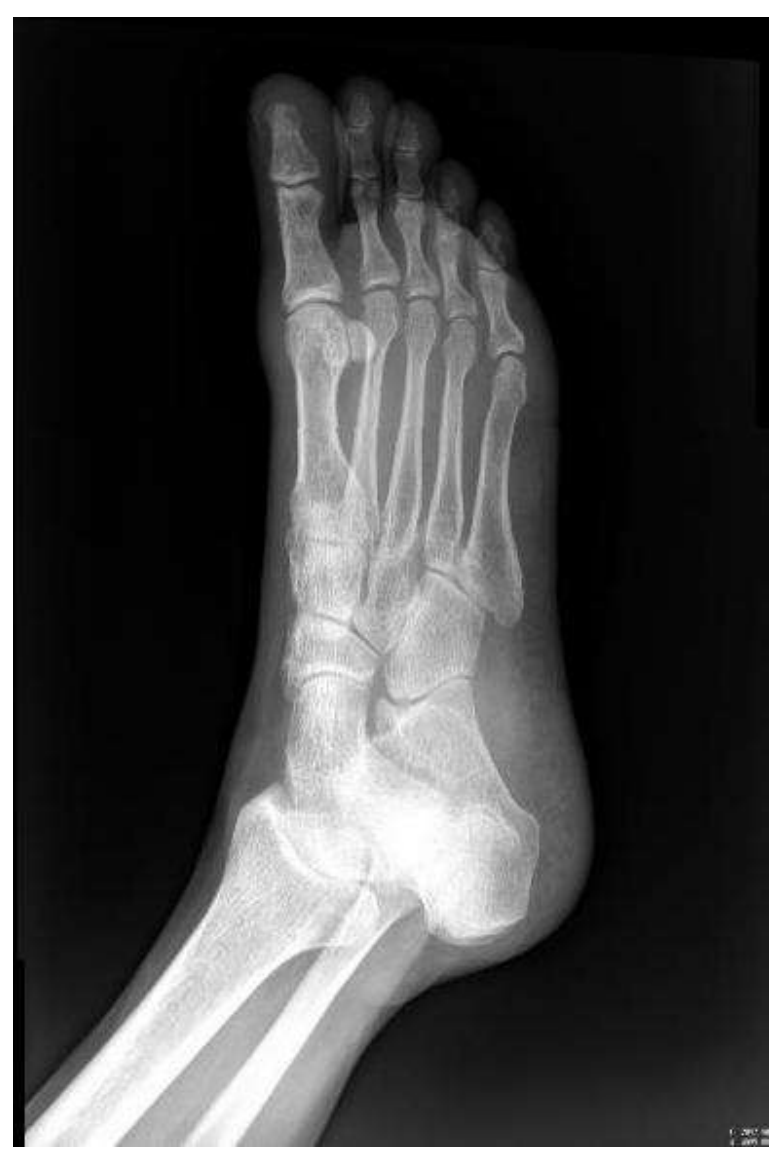

Figure 3. Final leg mosaicked image.

\begin{tabular}{|c|c|c|c|c|c|c|c|}
\hline Detector & $\begin{array}{c}\text { Image 1 } \\
\text { Feature } \\
\text { Points }\end{array}$ & $\begin{array}{c}\text { Image 2 } \\
\text { Feature } \\
\text { Points }\end{array}$ & $\begin{array}{c}\text { Time in } \\
\text { (sec) for } \\
\text { Image 1 }\end{array}$ & $\begin{array}{c}\text { Time in } \\
(\mathbf{s e c}) \\
\text { Image 2 }\end{array}$ & $\begin{array}{c}\text { Matching } \\
\text { Percentage }\end{array}$ & PSNR & MSE \\
\hline $\begin{array}{c}\text { Harris Corner } \\
\text { Detector }\end{array}$ & 48 & 52 & 0.74 & 0.76 & 10 & 5.67 & 1.67 \\
\hline SURF & 345 & 309 & 0.693 & 0.707 & 12.5 & 9.66 & 0.788 \\
\hline $\begin{array}{c}\text { Proposed } \\
\text { SIFT }\end{array}$ & 135 & 87 & 0.612 & 0.590 & 18 & 12.777 & 0.673 \\
\hline
\end{tabular}

Table 1: Performance Analysis of feature extraction detectors for the images 


\section{CONCLUSION}

In this work we proposed scale-invariant feature transform (SIFT) for mosaicking x-ray images as a local feature point extractor which uses difference of Gaussian (DOG) and invariant to orientation and scale. Based on the location relationship of x-ray images, random sample consensus (RANSAC) is incorporated to remove the effect of mismatched point pairs in x-ray images and to generate the panoramic view. The performances of the system are computed by using structural and time constraint parameters and are compared with different feature detection techniques. The experimental results shows that combing of SIFT and RANSAC yields less processing time.

\section{REFERENCES}

[1] XingyuDa, XialiWang, Xin Yu and Lihong Wang, "Bridge Surface Damage Image Stitching Method Based on SURF Algorithm ”, 20th COTA International Conference of Transportation, August, 2020.

[2] CansinYildiz, “An Implementation on Recognizing Panoramas", Department of computer engineering, Bilkent University, Ankara, Turkey, 2005. https://www.cs.bilkent.edu.tr/

[3] Dun Li; Dezhi Han; Xiaofeng Zhang; Li Zhang, "Panoramic image mosaic technology based on SIFT algorithm in power monitoring",6th International Conference on Systems and Informatics (ICSAI), 2019.

[4] Jun Hui Gong, Jun Hua Zhang, Zhen Zhou An, Wei Wei Zhao and Hui-Min Liu, "An approach for X-ray image mosaicing based on Speeded-Up Robust Features," International Conference on Wavelet Active Media Technology and Information Processing (ICWAMTIP), Chengdu, pp.432.435,2012.

[5] R. Botan, K. F. Coco and K. S. Komati, "Implementation of an Image Stitching Algorithm to a Low-Cost “ Digital Microscope", $30^{\text {th }}$ SIBGRAPI Conference on Graphics, Patterns and Images ( SIBGRAPI), Niterio, pp 285-291,2017, https://doi.org/ 10.1109/ICIHT.2017.7899142

[6] Ahmad P.Tafti, AhmadrezaBaghaie, Andrew B.Kirkpatrick, Jessica D. Holz, Heather A. Owen, “ A Comparative Study on applications of SIFT, SURF,BRIEF, and ORB for 3D surface reconstruction of electron microscopy images", Computer Methods in Biomechanics and Biomedical Engineering: Imaging \& Visualization, Volume 6, 2018-Issue 1.

[7] Sidong Zhang, Li and Dali Yin, "Image Matching Research Based on Improved SIFT Algorithm “, International Conference on Video, Signal, and Image Processing, 2019.

[8] Lowe, D. “ Distinctive Image Features from. Int. J. Comput. Vis”. 2004, 60, 91-110. [CrossRef]

[9] Xing, C.Huang, J. “ An Improved Mosaic Method Based on SIFT Algorithm for UAV Sequence Images ”. In proceedings of the 2010 International Conference on Computer Design and Applications, Qinhuangdao, China, 25-27 June 2010; Volume 1, pp. 414-417. [CrossRef]

[10] Yan,K; Sukthakar,R. PCA-SIFT: A More Distinctive Representation for Local Image Descriptors. In 
proceedings of the 2004 IEEE Computer Society Conference on Computer Vision and Pattern Recognition, 2004. CVPR 2004, Washington, DC, USA, 27 June-2 July 2004; Volume 2, pp. 506-513. [CrossRef]

[11] Ye, M; Androutsos, D. Robust Affine Invariant Region-Based Shape Descriptors: The ICA Zernike Moment shape Descriptor and the Whitening Zernike Moment Shape Descriptor. IEEE Signal Process. Lett 2009, 16, 877-80 [CrossRef].

[12]Samsudin S, Adwan,S., Arof,H.et al. J, “ Development of Automated Image Stitching System for Radiographic Images", Journal of digital Imaging, volume 26, Issue 2, pp. 361-370, April 2018.

[13] M. Dawood, C.Cappelle, M. E. El Najjar, M. Khalil and D. Pomorski, "Harris, SIFT and SURF features Comparison for vehicle localization based on virtual 3D model and camera", $3{ }^{\text {rd }}$ International Conference on Image processing Theory, Tools and Applications (IPTA), Istanbul, pp 307-312, 2012.

[14] Singla, Savita and Reecha Sharma, “ Medical Image Stiching Using Hybrid of SIFT \& SURF Techniques," International Journal of Advanced Research in Electronics and Communication Engineering (IJARECE) Volume 3, Issue 8, August 2014.

[15] Ebtsam Adel, Mohammed Elmogy and HazemElbakry " Image stiching system based on ORB Feature-Based Technique and Compensation Blending", International Journal of Advanced Computer Science and Applications, Vol. 6, No 9, pp. 55-62, 2015.

[16] Zhai. Y, Zeng.L, “ A SIFT Matching Algorithm Based on Adaptive Contrast Threshold ”, In Proceeding of the 2011 International Conference on Consumer Electronics, Communications and Networks(CECNet), XianNing, China, 16-18 April 2011, pp 1934-1937. [CrossRef]

[17] Wang, W. Hong, J. Tang, Y.P." Image Matching for Geomorphic Measurement Based on SIFT and RANSAC Methods", In Proceedings of the 2008 International Conference on Computer Science and Software Engineering, Hubei, China, 12-14 December 2008; pp 317-320. [CrossRef]

[18] Zhang, Y.H. Jin, X. Wang, Z.J. A New Modified Panoramic UAV Image Stiching Model Based on the GASIFT and Adaptive Threshold Method Memeticcomput. 2017, 9,231-244. [CrossRef]

[19] Fischler, M.A.Bolles, R.C. Random Sample Consensus: A Paradigm for Model Fitting with Applications to Image Analysis and Automated Cartography. Commum, ACM 1981, 24, 381-395. [CrossRef].

[20] Sattler T; Leibe B; Kobbelt L. SCRAAMSAC: Improving RANSAC's efficiency with a Spatial Consistency Filter. IN Proceedings of the 2009, IEEE $12^{\text {th }}$ International Conference on Computer Vision (ICCV), Kyoto, Japan, 29 September-2 October 2009; pp. 2090-2097. [CrossRef]

[21] Bhattacharya P; Gavilova. M. "Improving RANSAC Feature Matching with Local topological Information". In Proceedings of the 2012, Ninth International Symposium on Voronoi Diagrams in Science and Engineering (ISVD 2012), New Brunswick, NJ, USA, 27-29 June 2012; pp 17-23 [CrossRef] 